\title{
Particle Discriminator Interface for Nanoflow ESI-MS
}

\author{
Bradley B. Schneider, Vladimir I. Baranov, Hassan Javaheri, \\ and Thomas R. Covey \\ MDS SCIEX, Concord, Ontario, Canada
}

\begin{abstract}
An atmosphere to vacuum interface was designed to exploit the different mobility and momentum characteristics of ions, and charged and neutral particles in electrospray ionization-mass spectrometry. The purpose of this device is to transmit with high efficiency the ions created at atmospheric pressure into the mass analyzer and to deflect the large charged and neutral particles prior to entrance into the vacuum system, thereby maintaining system cleanliness and stability. This interface is particularly suitable for low flow rate electrospray ionization-mass spectrometry where the close proximity of the electrospray emitters to the vacuum entrance, and near total consumption of the entire spray, leads to the production of large quantities of non-desolvated droplets and large charged and neutral particles. The improvement involves the application of potential gradients to a particle discriminator space located between the gas restricting ion entrance orifice of the mass spectrometer and the exit of a heated laminar flow chamber to divert large particles from the gas conductance limiting orifice. A counter-current flow of drying gas is used to deflect neutral particles and solvent vapor. Two stages of desolvation are achieved with the combined effects of the curtain gas and heated laminar flow chamber. This enhances the efficiency of desolvation and ion production, and stabilizes the resulting ion current under a wide variety of solvent compositions. In addition, this system eliminates the problems associated with the boiling of solution in nanospray tips when operated in close proximity to a heated mass spectrometer inlet. The particle discriminator interface gives approximately a 2-fold improvement in ion count rates, and a 3-fold improvement in stability (as measured by the signal relative standard deviation). (J Am Soc Mass Spectrom 2003, 14, 1236-1246) (c) 2003 American Society for Mass Spectrometry
\end{abstract}

$\mathrm{E}$ lectrospray ionization is a powerful source of gas phase ions for mass spectral analysis. It is amenable to operation over a wide range of solvent compositions [1-3] and flow rates [4-7]. The desire to couple electrospray mass spectrometry with high flow separation techniques such as liquid chromatography (LC) has resulted in the development of various interfaces for high flow rate ESI-MS. Typical solution flow rates for LC-MS are on the order of 100-2000 $\mu \mathrm{L} / \mathrm{min}$. It has been common practice to split the LC flow, to reduce the volume of sample entering the electrospray source region [8], but recently new ion sources have been developed that allow stable operation with solution flow rates of greater than $2 \mathrm{~mL} / \mathrm{min}$ [9]. The development of high flow rate electrospray ion sources has primarily been driven by the LC market because of the lack of LC systems available for routine operation at lower solution flow rates. More recently, there has been an increased focus on lower flow rate electrospray ion

Published online August 26, 2003

Address reprint requests to Dr. B. B. Schneider, MDS SCIEX, 71 Four Valley Drive, Concord, Ontario L4K 4V8, Canada. E-mail: bradley. schneider@sciex.com sources due to higher ionization efficiency and lower sample consumption [7].

Mass spectrometer interface design can be very important for stable operation of ESI-MS systems at flow rates of less than $1 \mu \mathrm{L} / \mathrm{min}$. One type of ESI-MS interface incorporates a heated capillary inlet that provides thermal desolvation of droplets, while also limiting the gas conductance into the vacuum system of the mass spectrometer $[10,11]$. The temperature within the capillary and the residence time for droplets both control the extent of desolvation. A similar interface has also been described in which the heated capillary may have different inlet and exit apertures [12]. In both cases, charged droplet desolvation is promoted by heat alone and there is no discrimination between ions, neutrals, and large charged particles.

An alternate interface has a counter-current flow of "curtain gas" to aid in desolvation of droplets generated within an electrospray ion source and to sweep away unwanted neutral species from the entrance aperture $[7,13]$. The high electric field between an electrospray capillary and mass spectrometer inlet causes charged droplets to be propelled through the countercurrent gas flow, facilitating desolvation prior to arrival 
at the inlet orifice for the vacuum system. The curtain gas can be quite effective for desolvation purposes, provided that the initial droplet size is small. When the curtain gas is maintained at room temperature, this method is often referred to as "cold" desolvation and is frequently incomplete if the initial droplet size is not sufficiently small. The curtain gas repels charged particles to some extent but in the case of nanoflow ESI, where the emitters are positioned very close to the vacuum entrance, the combined effect of the attractive fields and vacuum flow draws the majority of them into the region of the aperture or into the vacuum system.

This paper describes a new interface for nanoflow ESI-MS. The new particle discriminator interface (PDI) is designed to improve performance by providing two stages of unwanted particle and solvent removal and two stages of desolvation. In addition to the curtain gas acting as a neutral particle and solvent deflector, a region is established in front of the gas conductance limiting ion entrance orifice where electric fields and bending laminar gas flow streamlines are used to extract charged particles from a gas flow containing ions and charged particles. Desolvation is effected by both the curtain gas and a heated laminar flow chamber directing the ion, particle, and gas stream into the discriminator region. In this fashion the functions of desolvation, particle discrimination, and vacuum interfacing are separated. Several other benefits accrue such as the desolvation heat is remote from the ESI emitters improving their stability for nanoflow ESI-MS and the electric fields and physical position of the emitters become less critical due to the large acceptance aperture of the laminar flow chamber.

\section{Experimental}

Samples of glufibrinopeptide (Sigma Chemical Co., St. Louis, MO) were prepared at $0.25 \mu \mathrm{M}$ in water acidified with $0.1 \%$ formic acid (Aldrich, Milwaukee, WI). Taurocholic acid (Sigma Chemical Co.) was prepared at 12 $\mathrm{pmol} / \mu \mathrm{L}$ in 50/50 water/acetonitrile (J. T. Baker, Phillipsburg, NJ) with $2 \mathrm{mM}$ ammonium acetate (Caledon Laboratories Ltd., Georgetown, ON). Myoglobin (Sigma Chemical Co.) was prepared at 2.4 pmole/ $\mu \mathrm{L}$ in $50 / 50$ water/acetonitrile with $0.1 \%$ acetic acid (Aldrich, Milwaukee, WI). 5-Fluorouracil (Sigma Chemical Co.) was prepared at $250 \mathrm{pg} / \mu \mathrm{L}$ in 50/50 water/acetonitrile with $2 \mathrm{mM}$ ammonium acetate. Reserpine (Sigma Chemical Co.) was prepared at $100 \mathrm{pg} / \mu \mathrm{L}$ in $70 / 30$ acetonitrile/ water with $2 \mathrm{mM}$ ammonium acetate and $0.1 \%$ formic acid. Cytochrome $c$ (Sigma Chemical Co.) was prepared at $5 \mathrm{mg} / \mathrm{mL}$ in $50 \mathrm{mM}$ ammonium bicarbonate (BDH Chemicals, Toronto, ON), $\mathrm{pH}$ adjusted to 8.5 with ammonium hydroxide (Fisher Scientific, Nepean, ON). Trypsin (Promega, Madison, WI) was added to the sample with a ratio of approximately 20:1 protein: trypsin. The digestion was carried out for $4 \mathrm{~h}$ at $37^{\circ} \mathrm{C}$. The digested protein was diluted to approximately 12 $\mathrm{pmol} / \mu \mathrm{L}$ in $50 / 50$ water/acetonitrile with $0.1 \%$ formic acid.

Tapered fused silica sprayer tips $(360 \mu \mathrm{m}$ o.d. tapered to $15 \mu \mathrm{m}$ i.d.) from New Objective (Woburn, MA) were connected to fused silica transfer capillaries (Polymicro Technologies, Phoenix, AZ) within a homebuilt microion spray unit. For very low flow rate experiments, nanoelectrospray tips from New Objective were used (1-2 $\mu \mathrm{m}$ i.d. at the tip). A low dead volume connection tee (Valco Instruments Co., Houston, TX) was fastened into the microion spray assembly. A modified Protana (Odense, DK) ion source was used to mount the sprayer and dual CCD cameras in front of the entrance aperture of the mass spectrometer. For microion spray experiments, sample was provided to the sprayer at a flow rate of $500 \mathrm{~nL} / \mathrm{min}$ with a syringe pump from Harvard Apparatus (South Natick, MA). The electrospray potential was applied directly to the stainless steel microion spray assembly to generate a liquid junction within the conductive connection tee. The tapered sprayer tip protruded approximately $7 \mathrm{~mm}$ from the microion spray head. The microion spray assembly was drilled so that a coaxial sheath gas could be introduced through a gas tube surrounding the tapered sprayer tip. The i.d. of the gas tube was bored out to $500 \mu \mathrm{m}$ leaving an annular space of $70-90 \mu \mathrm{m}$ around the fused silica capillary tip (360 $\mu$ m o.d., 320 $\mu \mathrm{m}$ with the polyimide coating burned off). For nanoelectrospray experiments, sample was provided at a flow rate of $50 \mathrm{~nL} / \mathrm{min}$ or less, and no sheath gas was provided.

The mass spectrometer used for these studies was a modified API 3000 from MDS SCIEX (Concord, ON). The interface region was modified to incorporate a heated laminar flow chamber prior to the orifice. The orifice plate was grooved to permit attachment of a 1 $\mathrm{mm}$ thick Teflon spacer (6 $\mathrm{mm}$ aperture) and heated stainless steel chamber. Dual cartridge heaters (Whatlow, St. Louis, MO) were attached to an aluminum sleeve on the outside surface of the chamber. The commercial connector for attachment of the curtain plate potential was removed to allow the feedthroughs from the cartridge heater to be inserted and sealed through the source housing. The heater feedthroughs were attached to a Variac heater (Superior Electric, Bristol, CT) to give a maximum heater temperature of approximately $250{ }^{\circ} \mathrm{C}$. The heated chamber was composed of a $13 \mathrm{~mm}$ long, straight $1 \mathrm{~mm}$ channel drilled into an $8 \mathrm{~mm}$ o.d. cylinder. For experiments with longer heated chambers, pieces of stainless steel syringe tubing (1/8 inch o.d., $1 \mathrm{~mm}$ i.d.) of various lengths were press-fitted into stainless steel chambers. Four screws were used to hold the chamber and Teflon spacer onto the orifice plate. The commercial curtain plate was replaced with a flat-faced curtain plate that had an 8.5 $\mathrm{mm}$ diameter aperture. A spring was used to ensure that the chamber and curtain plate were maintained at the same potential, as controlled by an external power supply (Bertan Associates Inc., 0-1 kV, Hicksville, NY). 
(a)
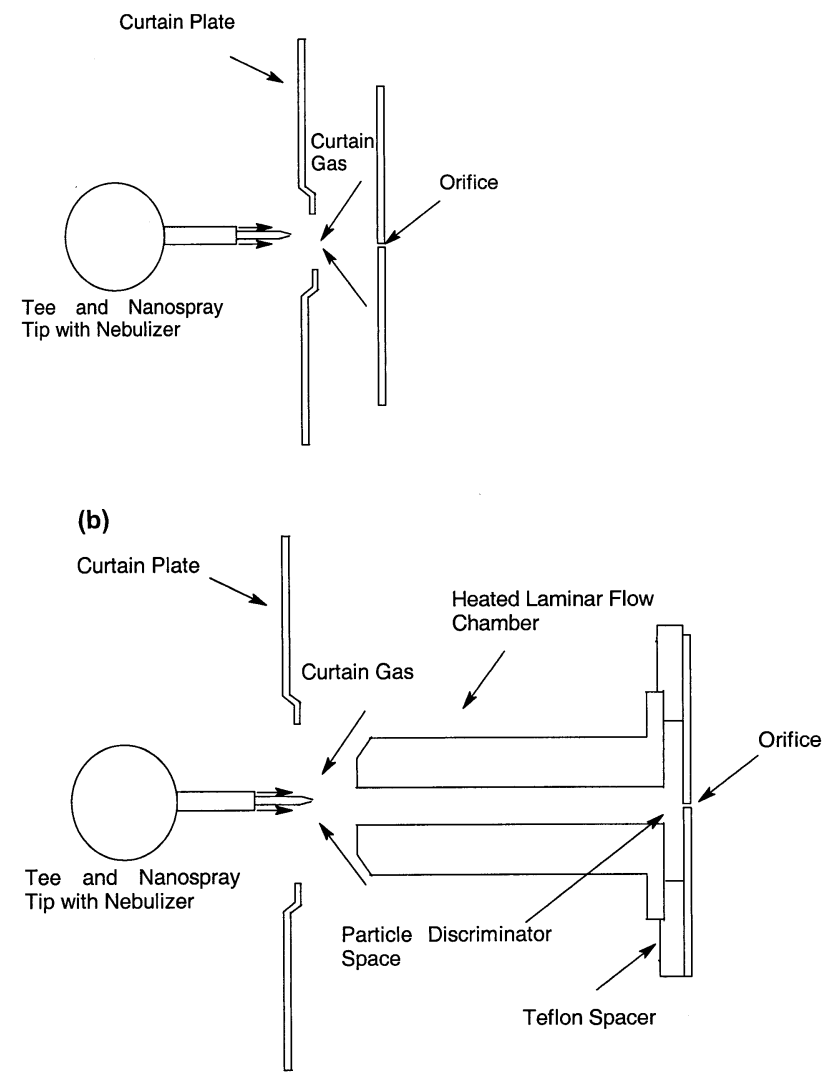

Figure 1. (a) Schematic of a standard interface for electrospray ionization that incorporates a counter-current flow of curtain gas. (b) Schematic of an improved interface that incorporates a heated laminar flow chamber and a particle discriminator space into the atmospheric pressure region between a curtain plate and orifice plate.

For some experiments, the curtain plate was removed and no counter-current gas flow was provided. Nitrogen and compressed air from Praxair (Mississauga, $\mathrm{ON}$ ) were used as the curtain and sheath gases.

\section{Results}

The standard API 3000 interface and the particle discriminator interface are shown in Figures $1 \mathrm{a}$ and $\mathrm{b}$, respectively. Nanoflow ESI with the standard interface typically involves positioning the tapered sprayer tip within a few millimeters of the curtain plate. Ions and charged droplets from the sprayer travel through the counter-current gas flow as they enter a $3 \mathrm{~mm}$ aperture in the curtain plate. "Cold" desolvation occurs during transit through the $3 \mathrm{~mm}$ space between the curtain plate and the $0.25 \mathrm{~mm}$ orifice. Ions and charged droplets within the vicinity of the orifice are drawn into the vacuum system of the mass spectrometer with a portion of the curtain gas that is adjusted to have strong counter-current flow. However, when operating within the flow regime of approximately $100-1000 \mathrm{~nL} / \mathrm{min}$, the "cold" desolvation capabilities of the curtain gas can be insufficient for samples prepared in predominantly aqueous solvent. Increasing either the curtain gas flow, or the sprayer-curtain plate spacing to compensate for this typically attenuates ion count rates.

Figure $1 \mathrm{~b}$ shows a schematic of the improved interface, with a heated laminar flow chamber and a particle discriminator space inserted into the atmospheric pressure region between the curtain plate and orifice plate. With this configuration, the sprayer is typically operated flush with, or inside the aperture in the curtain plate. The heated chamber is sealed onto the orifice plate with a $1 \mathrm{~mm}$ thick Teflon spacer, allowing the vacuum draw from the orifice to establish laminar flow conditions down the $1 \mathrm{~mm}$ bore of the $13 \mathrm{~mm}$ chamber. The large internal diameter of the chamber relative to the orifice $(4 \times$ larger) ensures that there is no significant pressure drop down the length of the heated chamber. In fact, the chamber length could be increased by a factor of 6 with no change in the downstream pressure readings within the mass spectrometer because the flow is restricted by the orifice. The inner diameter of the spacer creates a particle discriminator space. In this region, where the laminar gas flow streamlines bend to enter the orifice, large charged particles can be extracted from the gas flow into the orifice. Ions follow the gas streamlines through the orifice, whereas larger charged particles (solid material or residual droplets) have sufficient momentum to break free of the gas streamlines. The presence of a strong electric field within the particle discriminator space (approximately $4000 \mathrm{~V} / \mathrm{cm}$ ) directs the particles onto the orifice plate in the region surrounding the aperture. The confluence of the curtain gas, which also splits into outward and inward directed flows, and sheath gas generates a region of little or no flow within the $3 \mathrm{~mm}$ space (curtain gas chamber) between the curtain plate and the front of the heated chamber. Ions and charged droplets within this region of "cold" desolvation are drawn into the heated chamber entrance with the inward flow of a portion of the curtain gas. Ions and charged droplets undergo "hot" desolvation as they travel through the heated chamber towards the orifice. This configuration allows the user to operate effectively with a lower curtain gas flow and a smaller spacing between the sprayer and inlet, as opposed to the standard interface.

The gas flow through the heated chamber is dictated by the gas throughput of the orifice $(\mathrm{G})$. The relationship between the gas throughput and orifice diameter is shown in eq 1 (assuming nitrogen as the curtain gas);

$$
G=0.445 n_{0} a_{0} D_{0}^{2}
$$

where $n_{0}$ is the number density within the source $(2.5 \times$ $10^{19}$ molecules $\left./ \mathrm{cm}^{3}\right), a_{0}$ is the speed of sound in the source $\left(3.50 \times 10^{4} \mathrm{~cm} / \mathrm{s}\right)$ and $D_{0}$ is the orifice diameter $(0.025 \mathrm{~cm})$ [14]. From eq 1, the gas throughput for the room temperature chamber is $2.53 \times 10^{20}$ molecules/s, corresponding to an average gas flow speed of $12.9 \mathrm{~m} / \mathrm{s}$ 
through the chamber. This gives a residence time of approximately $1 \mathrm{~ms}$ for ions or charged droplets within the chamber.

The Reynolds number $\left(\mathrm{N}_{\mathrm{Re}}\right)$ is a common parameter that can be used to assess the properties of various flows. Eq 2 gives an expression for the Reynolds number,

$$
N_{\mathrm{Re}}=\frac{\rho \nu d}{\mu}
$$

where $p$ is the gas density, $v$ is the average flow velocity, $d$ is the channel diameter, and $\mu$ is the viscosity of the gas [15]. Assuming a $13 \mathrm{~m} / \mathrm{s}$ nitrogen gas flow through a $1 \mathrm{~mm}$ channel, the Reynolds number is determined to be less than 1000, ensuring that conditions of laminar flow prevail through the chamber. Eq 2 demonstrates that larger internal diameter chambers may be used without generating turbulent flow $(2 \times$ larger diameter countered by $4 \times$ smaller gas flow velocity) through the chamber. It is desirable to avoid conditions of turbulent flow because its unstable nature promotes ion diffusional losses to the walls of the chamber [15] and contributes to overall signal instability. The fraction of ions lost by diffusion to the walls of the chamber can be approximated from eq 3 [16];

$$
I=I_{0} \exp \left[-2.405^{2}\left(\frac{2 l}{d}\right)^{2} \frac{k T}{q U_{e f f}}\right]
$$

where $I$ is the transmitted ion current, $I_{0}$ is the entrance ion current, $l$ is the length of the chamber, $d$ is the diameter of the chamber, $k$ is Boltzmann's constant, $T$ is the temperature, $q$ is the ion charge, and $U_{\text {eff }}$ is the effective potential difference down the length of the chamber. Eq 3 gives the estimated ion diffusional loss for transport through a capillary with different potentials on either end, and no gas flow. Since ion mobility through this chamber is controlled by the gas flow speed, the effective potential difference down the length of the chamber (or the potential difference required to generate the same ion mobility in the absence of the gas flow) is given by eq 4;

$$
U_{e f f}=\left(\frac{\nu}{K}\right) l
$$

where $v$ is the average gas flow speed through the chamber and $K$ is the ion mobility. For a typical ion mobility of $1 \mathrm{~cm}^{2} / \mathrm{Vs}$ [17], and $13 \mathrm{~m} / \mathrm{s}$ gas flow speed, the effective potential difference is $1690 \mathrm{~V}$. Using an $\mathrm{l} / \mathrm{d}$ ratio of 13 and a temperature of $295 \mathrm{~K}$, eq 3 gives a transport efficiency of approximately $94.6 \%$ for singly charged ions. It is important to note that eq 3 predicts that the transport efficiency increases for multiply charged ions due to increased mobility through the chamber. In reality, there is no potential difference down the length of the heated chamber, so the transport efficiency for multiply charged ions should be similar to the above value calculated for singly charged ions providing that the transverse diffusion coefficient is similar. Increasing the temperature to $423 \mathrm{~K}$, the transport efficiency drops to approximately $92 \%$ of the initial ion current. Eq 3 demonstrates the importance of minimizing the $l / d$ ratio to minimize diffusional losses within the chamber. Experiments were conducted to verify the validity of the above calculation. Eq 3 predicts that increasing the internal diameter of the chamber from 1 to $2 \mathrm{~mm}$, while maintaining a $13 \mathrm{~mm}$ length should have no effect on ion transmission efficiency through the chamber, independent from the vacuum interface requirements. The factor of four decrease in the $(2 l / d)^{2}$ term is countered by a factor of four decrease in the $U_{\text {eff }}$ term. Experiments conducted with 1 and 2 $\mathrm{mm}$ i.d. chambers have verified that ion count rates are very similar with the two designs. Further support for eq 3 can be generated by comparison of experimental count rates for ions using chambers with various lengths and a $1 \mathrm{~mm}$ i.d. Eq 3 predicts reductions in transmission efficiency for 23 and $33 \mathrm{~mm}$ chambers relative to a $13 \mathrm{~mm}$ chamber, assuming that there is no gas flow restriction. Experiments with a sample of $1 \mu \mathrm{M}$ bradykinin prepared in 50/50 water/methanol with $0.1 \%$ formic acid showed that the count rate decreased by factors of 1.3 and 1.7, respectively for the 23 and 33 $\mathrm{mm}$ chambers. Ion losses were slightly greater than that predicted by diffusion alone with the longer chambers. It is possible that this was due in part to sealing and alignment difficulties with the press-fitted centers of the longer chambers.

Ion transport through the heated chamber is completely dominated by the laminar gas flow. Figure 2 shows mass spectra acquired for a sample of 2.4 pmole $/ \mu \mathrm{L}$ myoglobin. The curtain plate was removed for the collection of these data, and the orifice potential was $180 \mathrm{~V}$. The electrospray potential was adjusted so that it was approximately $1600 \mathrm{~V}$ higher than the chamber potential. Figure 2 shows data collected with the chamber maintained at $500 \mathrm{~V}$, and with the chamber maintained at the same potential as the orifice $(180 \mathrm{~V})$. The spray potential was optimized for the +17 charge state $(m / z=998)$. The count rate for myoglobin ions was very similar regardless of the potential difference applied between the chamber and the orifice plate. Similar experiments were conducted with lighter ions such as deprotonated taurocholic acid (data not shown) to demonstrate that ion count rates were similar with the chamber maintained at the orifice potential or higher. These data show that the motion of ions through this region is largely dominated by the gas flow through the orifice. Ions are swept along within the laminar gas flow generated by the vacuum draw of the orifice. As an additional evidence for this conclusion, we observe that ion count rates decreased when the chamber was not sealed properly to the front of the orifice plate. However, the motion of larger charged particles was con- 


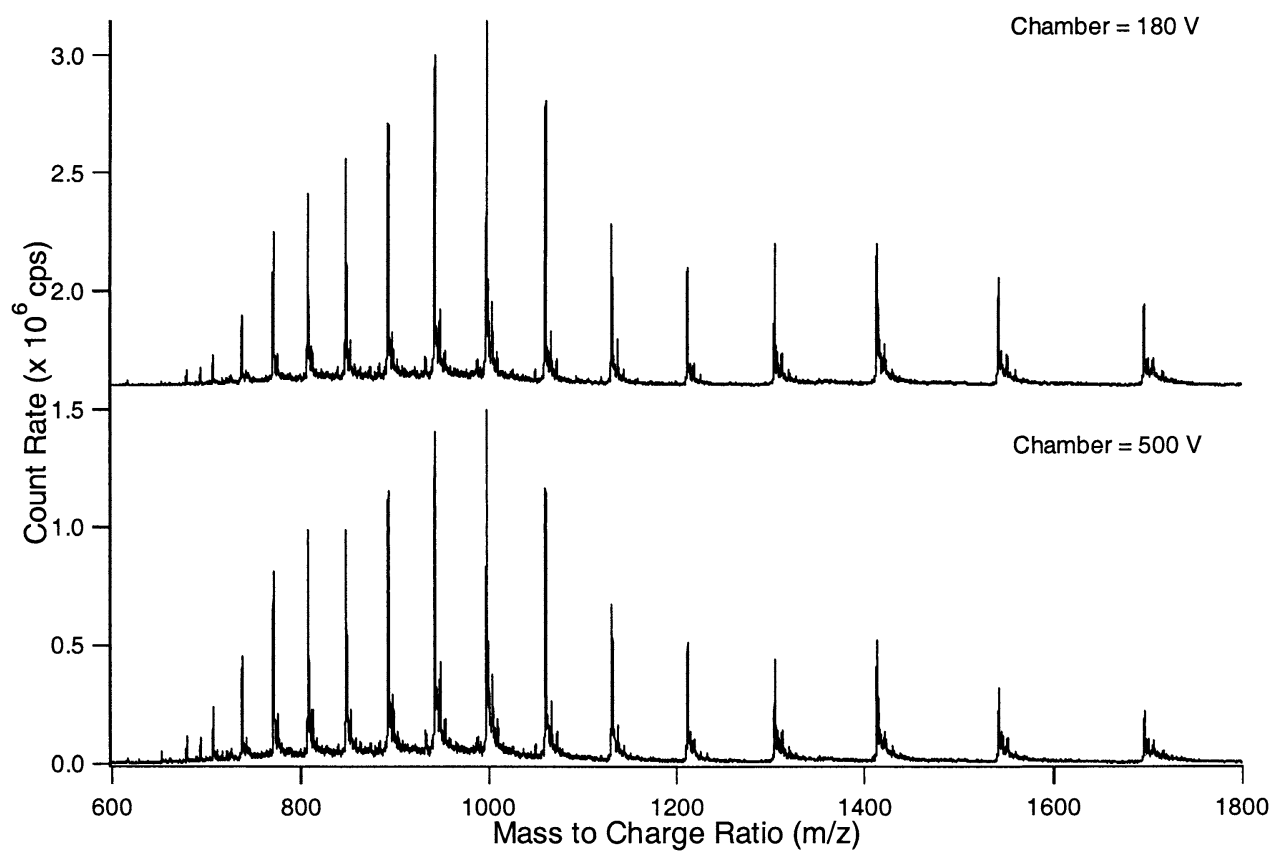

Figure 2. Mass spectra for a sample of myoglobin with the heated chamber maintained at 180 and $500 \mathrm{~V}$. The predominant peaks correspond to the +10 to +24 charge states of the protein, with $\mathrm{m} / \mathrm{z}$ values of $707,738,771,808,848,893,943,998,1061,1131,1212,1305,1413,1542$, and 1696.

trolled by both the gas flow and the electric field applied between the exit of the heated chamber and the orifice. In this region, the laminar gas flow streamlines exiting the $1 \mathrm{~mm}$ i.d. chamber bend to enter the $0.25 \mathrm{~mm}$ diameter orifice. Large particles carry sufficient momentum to break free of the gas streamlines. These large particles formed a deposit on the orifice plate surrounding the aperture, and the diameter of this deposit increased with the electric field in this region. Therefore, application of large electric fields (4000-5000 $\mathrm{V} / \mathrm{cm}$ ) within this region was advantageous because particulates were dispersed from the aperture to a greater extent, without affecting the count rate for ions. The incorporation of a particle discriminator region between the heated laminar flow chamber and orifice plate substantially improved the robustness and longterm stability with this system.

Experiments were conducted in which the inner diameter of the heated chamber bore was tapered from $1 \mathrm{~mm}$ at the inlet to approximately $300 \mu \mathrm{m}$ at the exit. Tapering the chamber did not increase ion count rates or stability with this interface. In fact, it lead to decreased robustness because the tapered chamber was prone to pluggage. In addition, the taper directed residual contamination directly onto the orifice, resulting in the need for much more frequent cleaning. Experiments were conducted where a sample of 0.25 $\mu \mathrm{M}$ glufibrinopeptide was sprayed for 17 consecutive hours with a tapered chamber. The chamber temperature was $70{ }^{\circ} \mathrm{C}$, and the gas flow rates were 0.8 and 2.0 $\mathrm{L} / \mathrm{min}$ for the sheath and curtain gases, respectively. The potentials were 2500,500 , and $80 \mathrm{~V}$ for the sprayer, curtain plate/chamber, and orifice, respectively. With the tapered chamber, particulates accumulated directly on the orifice, leading to a factor of 2 decrease in signal over $17 \mathrm{~h}$. Repeating this experiment with the nontapered chamber (i.d. $=1 \mathrm{~mm}$ ), particulates were deposited over a much larger area around the orifice, and there was no count rate reduction over the course of $17 \mathrm{~h}$. Tapering the heated chamber allowed the gas streamlines to bend much more gradually in the region prior to the orifice, thereby reducing the effectiveness of the particle discriminator.

The incorporation of the heated laminar flow chamber improved the nanoflow ESI performance of the mass spectrometer substantially for aqueous samples. Figure 3 shows the count rate stability for an aqueous sample of $0.25 \mu \mathrm{M}$ glufibrinopeptide run on the standard interface, the particle discriminator interface with no curtain or sheath gas, and the particle discriminator interface with both curtain and sheath gases. The solution flow rate was $500 \mathrm{~nL} / \mathrm{min}$ for all of the data presented in Figure 3. For the standard interface, the sprayer, curtain plate, and orifice potentials were 2000, 500 , and $75 \mathrm{~V}$, respectively. The sprayer was located within approximately $1 \mathrm{~mm}$ of the aperture in the curtain plate, and the curtain gas flow was provided at approximately $1.0 \mathrm{~L} / \mathrm{min}$. For the PDI, the potentials applied to the sprayer, curtain plate, heated chamber, and orifice were 1900, 500, 500, and $75 \mathrm{~V}$, respectively. The sprayer was inserted approximately $1 \mathrm{~mm}$ through the curtain plate, and the chamber was maintained at $205{ }^{\circ} \mathrm{C}$. The sheath and curtain gas flows were 0.6 and $1.6 \mathrm{~L} / \mathrm{min}$, respectively. The average count rate for doubly protonated peptide ions was 1.6, 1.4, and 0.74 million cps for the PDI with gases, PDI with no gases, 


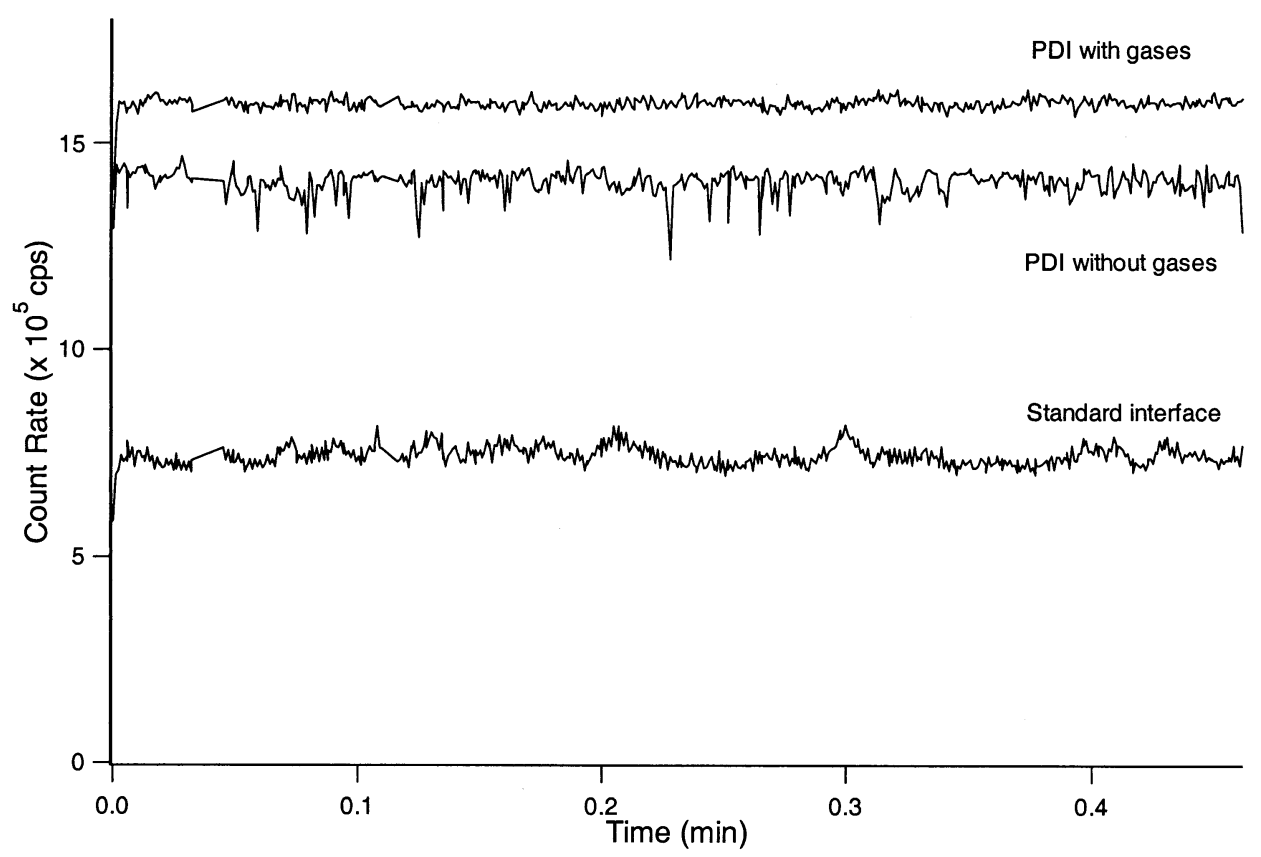

Figure 3. Count rate and stability comparisons for the standard interface and the PDI with and without curtain and sheath gases. The count rate for doubly protonated glufibrinopeptide ions $(\mathrm{m} / \mathrm{z}=$ 786) was monitored for an aqueous sample of $2.5 \times 10^{-7} \mathrm{M}$ peptide.

and standard interface. The count rate relative standard deviation was $0.94,2.1$, and $3.2 \%$, respectively, $(0.010$, 0.011 , and $0.015 \%$, respectively, predicted from count statistics). Although all methods are well above the count statistics limit, comparing the optimum results for the standard interface and the PDI, there was a 3.2 times improvement in stability, and a two times improvement in count rate for samples prepared in acidified water. These data reflect the improvements in count rate and stability for aqueous samples measured over a relatively short acquisition time (30 s). The stability improvement becomes even more significant when monitored over much longer time periods (hours or days) because the standard interface is more sensitive to variations in experimental conditions when spraying aqueous samples.

The particle discriminator interface also improved the performance of this system for samples prepared in solvents with a greater organic content. Figure 4 shows a comparison of mass spectra for a $100 \mathrm{pg} / \mu \mathrm{L}$ sample of reserpine prepared in 70/30 acetonitrile/water with 2 $\mathrm{mM}$ ammonium acetate and $0.1 \%$ formic acid. The data generated with the standard interface and the PDI are shown on the top and the bottom, respectively. For the

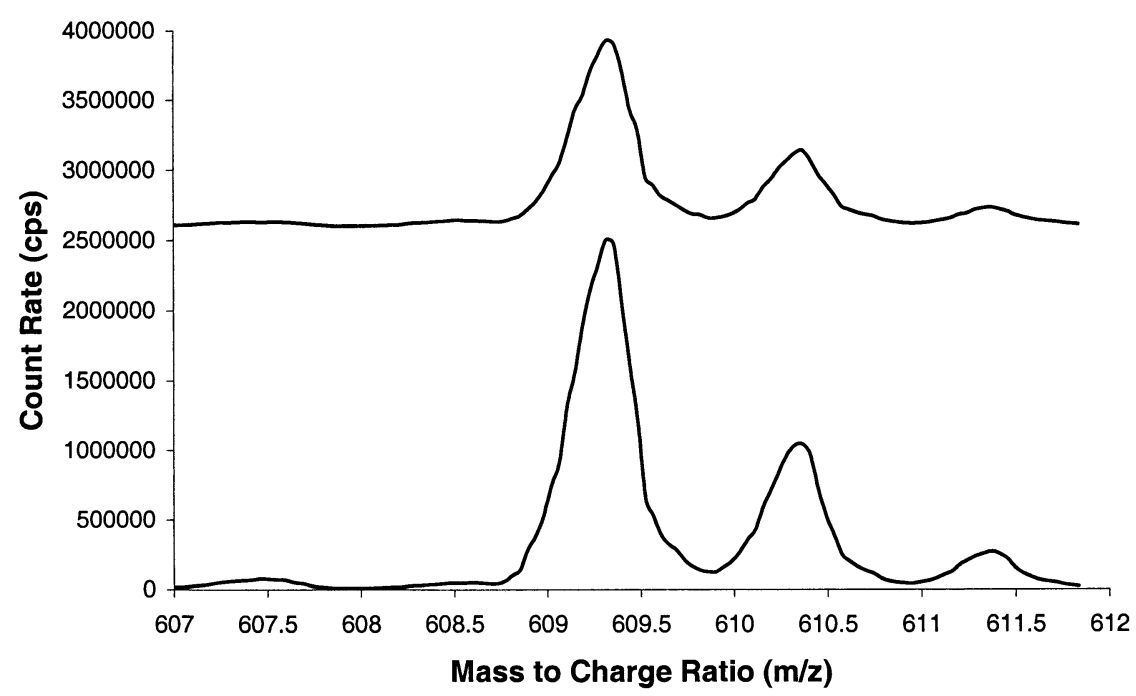

Figure 4. Comparison of mass spectra for a sample of reserpine using the standard interface (top) and the PDI (bottom). 
Table 1. Count rate and stability for deprotonated taurocholic acid with increasing distance between a microion spray tip and a heated laminar flow chamber

\begin{tabular}{llllllll}
\hline Sprayer-chamber spacing & $6 \mathrm{~mm}$ & $5 \mathrm{~mm}$ & $4 \mathrm{~mm}$ & $3 \mathrm{~mm}$ & $2 \mathrm{~mm}$ & $1 \mathrm{~mm}$ & $0 \mathrm{~mm}$ \\
Standard deviation $\left(\times 10^{4} \mathrm{cps}\right)$ & 4.6 & 4.7 & 5.0 & 5.0 & 5.7 & 7.1 & 8.1 \\
Average count rate $\left(\times 10^{6} \mathrm{cps}\right)$ & 2.50 & 2.59 & 2.57 & 2.70 & 2.73 & 3.03 & 3.02 \\
RSD $(\%)$ & 1.8 & 1.8 & 1.9 & 1.8 & 2.1 & 2.3 \\
\hline
\end{tabular}

standard interface, the potentials were 2200, 1000, and $80 \mathrm{~V}$ applied to the sprayer, curtain plate, and orifice plate, respectively. The sheath and curtain gases were 0.2 and $1.6 \mathrm{~L} / \mathrm{min}$, respectively. For the PDI, the potentials were 1400,500 , and $80 \mathrm{~V}$ applied to the sprayer, curtain plate/laminar flow chamber, and orifice, respectively. The laminar flow chamber was heated to 135 ${ }^{\circ} \mathrm{C}$, and the sheath and curtain gases were set to 0.6 and $1.6 \mathrm{~L} / \mathrm{min}$, respectively. The PDI increased the count rate for protonated reserpine ions by a factor of approximately 1.8. In addition, the signal stability (count rate RSD) improved by a factor of 1.7. Typical stability improvements with the PDI were on the order of approximately $1.5-2 \times$ for samples prepared in solvents with greater than $40 \%$ organic content.

The sheath gas serves two functions with the PDI. It helps to stabilize the electrospray process, but it also helps to cool the sprayer tip. When the chamber temperature is maintained around $200{ }^{\circ} \mathrm{C}$, the curtain gas flowing out through the curtain plate is hot enough to cause the solvent within nanoflow sprayer tips to boil. Boiling within the tapered tips causes the spray process to stop, and blackened salts build up to plug sprayers. The incorporation of a sheath gas prevents boiling, and also relaxes the sprayer-chamber positional requirements for nanoflow tips. Table 1 shows data collected for a sample of taurocholic acid. The data show the average count rate and stability for 10 measurements of the intensity for ions of deprotonated taurocholic acid as the separation between the sprayer and chamber changed from $0 \mathrm{~mm}$ (flush with the chamber inlet) to 6 $\mathrm{mm}$. Since the spacing between the curtain plate and chamber was $3 \mathrm{~mm}$, the sprayer was inserted $3 \mathrm{~mm}$ through the curtain plate aperture for the data at $0 \mathrm{~mm}$. For the data at $6 \mathrm{~mm}$, the sprayer was $3 \mathrm{~mm}$ outside of the aperture in the curtain plate. The electrospray potential decreased from approximately $-2000 \mathrm{~V}(6$ $\mathrm{mm}$ spacing) to approximately $-1400 \mathrm{~V}(0 \mathrm{~mm}$ spacing). In addition, the curtain and sheath gases were adjusted to optimize the count rate at each position. The count rates measured by the mass spectrometer were relatively insensitive to the sprayer to chamber spacing. There was a slight increase in count rate with very small spacing (less than $2 \mathrm{~mm}$ ) however, the count rate was less stable. The count rate and stability were reasonably constant with spacings of $3-6 \mathrm{~mm}$. These data are important because they demonstrate that extensive sprayer positional optimization is not necessary with this interface.

Figure 5 shows a comparison of negative ion mode mass spectra acquired for a $250 \mathrm{pg} / \mu \mathrm{L}$ sample of 5-fluorouracil (5-FU), prepared in 50/50 water/acetonitrile with $2 \mathrm{mM}$ ammonium acetate, with the chamber maintained at $74{ }^{\circ} \mathrm{C}, 133{ }^{\circ} \mathrm{C}$, and $205^{\circ} \mathrm{C}$. The sample

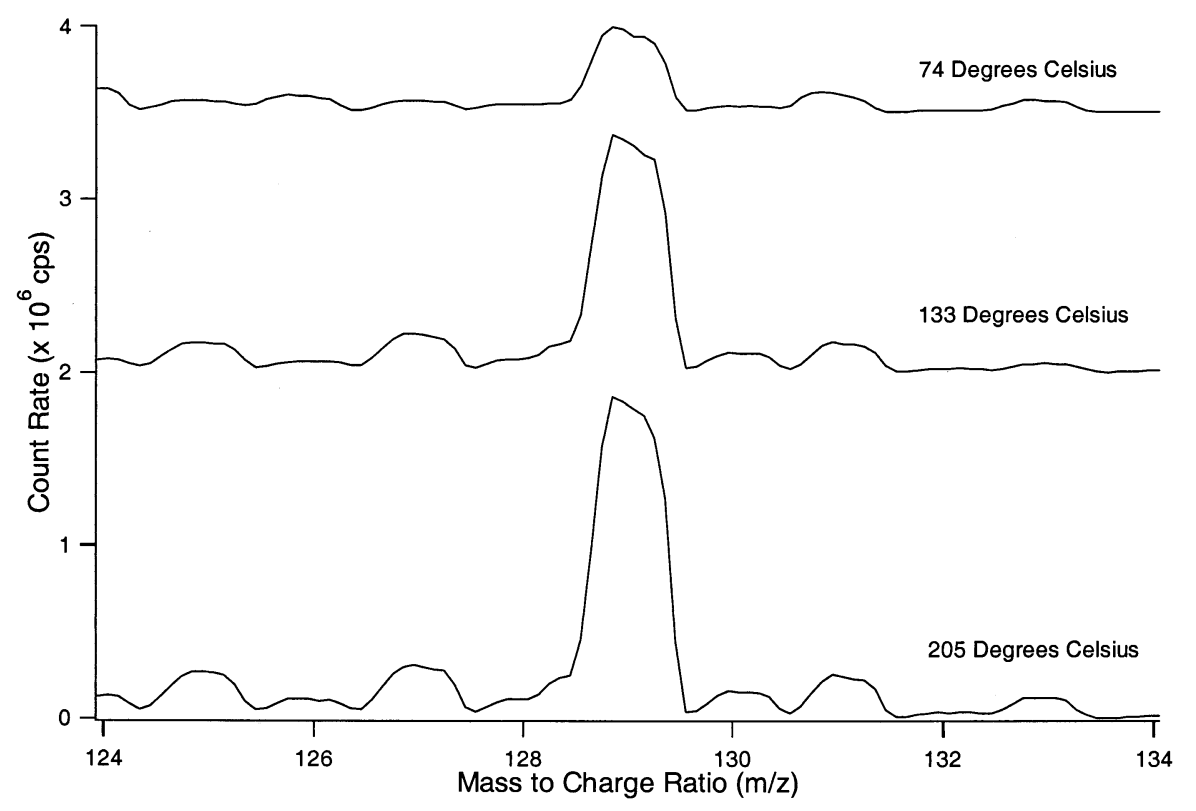

Figure 5. Comparison of negative ion mode mass spectra for a sample of 5-fluorouracil with the chamber maintained at $74{ }^{\circ} \mathrm{C}, 133{ }^{\circ} \mathrm{C}$, and $205^{\circ} \mathrm{C}$. 


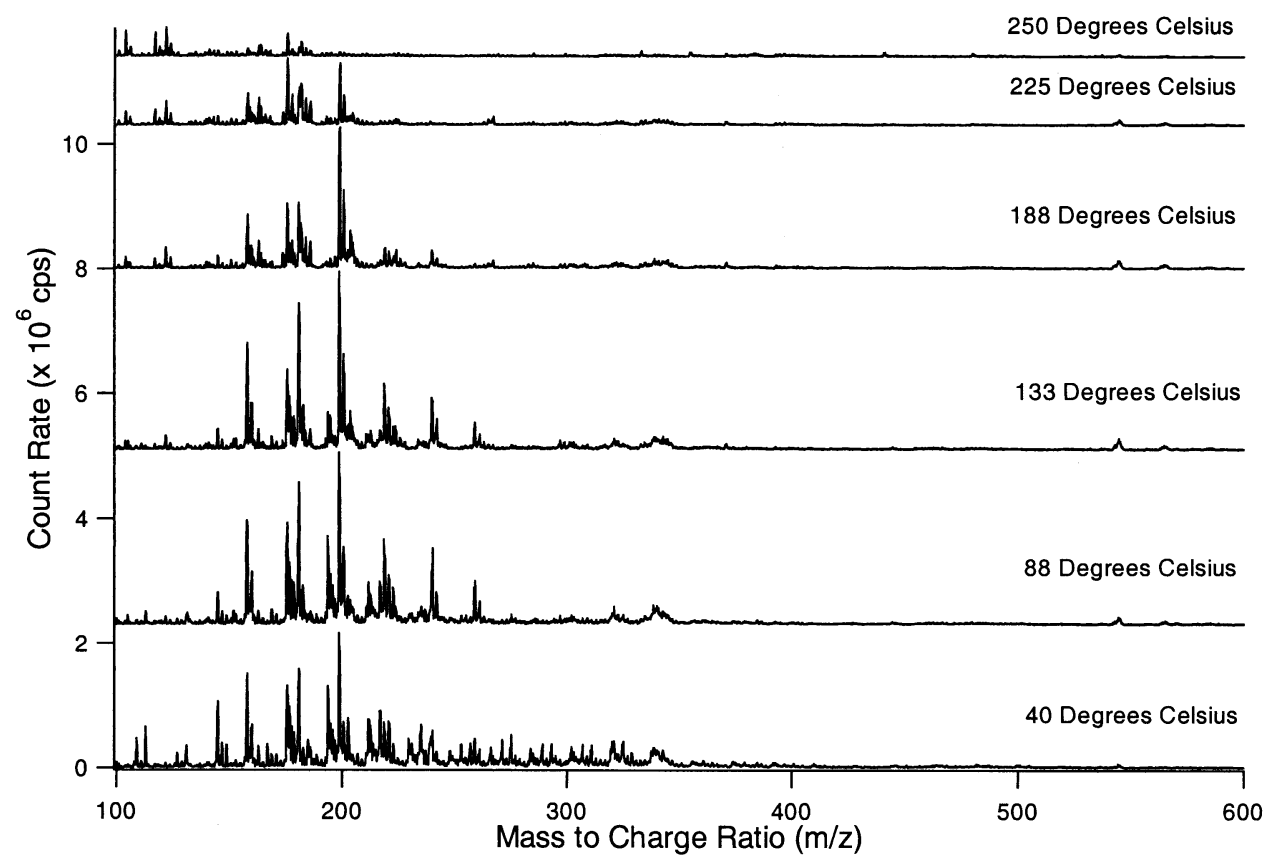

Figure 6. Comparison of the low mass chemical noise for a sample of glufibrinopeptide prepared in acidified water with the chamber maintained at $40{ }^{\circ} \mathrm{C}, 88^{\circ} \mathrm{C}, 133^{\circ} \mathrm{C}, 188^{\circ} \mathrm{C}, 225^{\circ} \mathrm{C}$, and $250{ }^{\circ} \mathrm{C}$.

flow rate was $500 \mathrm{~nL} / \mathrm{min}$ and the sprayer location was not changed during the experiment. The potentials applied to the sprayer, curtain plate, chamber, and orifice were $-1650,-500,-500$, and $-45 \mathrm{~V}$, respectively. The sheath and curtain gas flows were 0.2 and $1.6 \mathrm{~L} / \mathrm{min}$, respectively. Increasing the temperature of the chamber from $74{ }^{\circ} \mathrm{C}$ to $205{ }^{\circ} \mathrm{C}$ substantially improved the count rate for ions of deprotonated 5-FU (3.5 $\times$ improvement in ion count rate). These data demonstrate that even with a reasonably high curtain gas flow $(1.6 \mathrm{~L} / \mathrm{min})$, heating the chamber can be beneficial to performance when operating with solution flow rates on the order of $500 \mathrm{~nL} / \mathrm{min}$. Since all other parameters were constant for collection of these data, the improvement must be due to more efficient desolvation within the atmospheric pressure region prior to the ion sampling orifice. The count rate improvement with chamber heat was found to be somewhat compound dependent. Smaller gains were achieved with some other samples $(1.5 \times$ improvement with bradykinin prepared in 60/40 water/methanol with $1 \%$ acetic acid). For compounds with poor thermal stability, it was advantageous to use lower temperatures in combination with increased curtain gas flows.

A final benefit of incorporating a heated chamber into the interface region of a system equipped for low flow rate electrospray can be a decrease in the level of the chemical noise. When running at lower sample flow rates, it may be necessary to lower the curtain gas flow rate substantially to generate maximum ion signals. Under circumstances where the system is operated with a very low curtain gas flow or a small spacing between the sprayer and orifice, the intensity of low mass background peaks can become significant in the absence of "hot" desolvation. Figure 6 shows a comparison of the low mass background generated for a sample of $0.25 \mu \mathrm{M}$ glufibrinopeptide prepared in acidified water. The curtain gas flow was turned off for the collection of these data. The potentials were 2100, 500, and $100 \mathrm{~V}$ applied to the sprayer, chamber, and orifice, respectively. The chamber temperature was increased from approximately $40{ }^{\circ} \mathrm{C}$ to $250{ }^{\circ} \mathrm{C}$, resulting in substantial reduction in the count rate for the low mass background. In addition, the count rate for the peptide increased by a factor of two as the temperature increased from $40{ }^{\circ} \mathrm{C}$ to $133{ }^{\circ} \mathrm{C}$, and was relatively constant as the temperature increased to $250{ }^{\circ} \mathrm{C}$ (data not shown). These data demonstrate the importance of heating a nanoflow interface to lower the chemical noise when operating with a negligible or low curtain gas flow.

The particle discriminator interface was also found to be useful for operation at very low flow rates (50 $\mathrm{nL} / \mathrm{min}$ or less). Initial experiments were performed with no curtain gas or curtain plate. In this configuration, it was necessary to heat the chamber to eliminate solvent clusters from the spectra. However, heating the chamber above $100{ }^{\circ} \mathrm{C}$ resulted in the onset of boiling within the nanospray tips. For this mode of operation, a compromise had to be reached, where the sprayer was approximately $2 \mathrm{~mm}$ from the chamber entrance, and the chamber temperature was maintained at approximately $74{ }^{\circ} \mathrm{C}$. Nanoelectrospray was more effective when the curtain plate was reinstalled, and a curtain gas was used for "cold" desolvation of charged drop- 


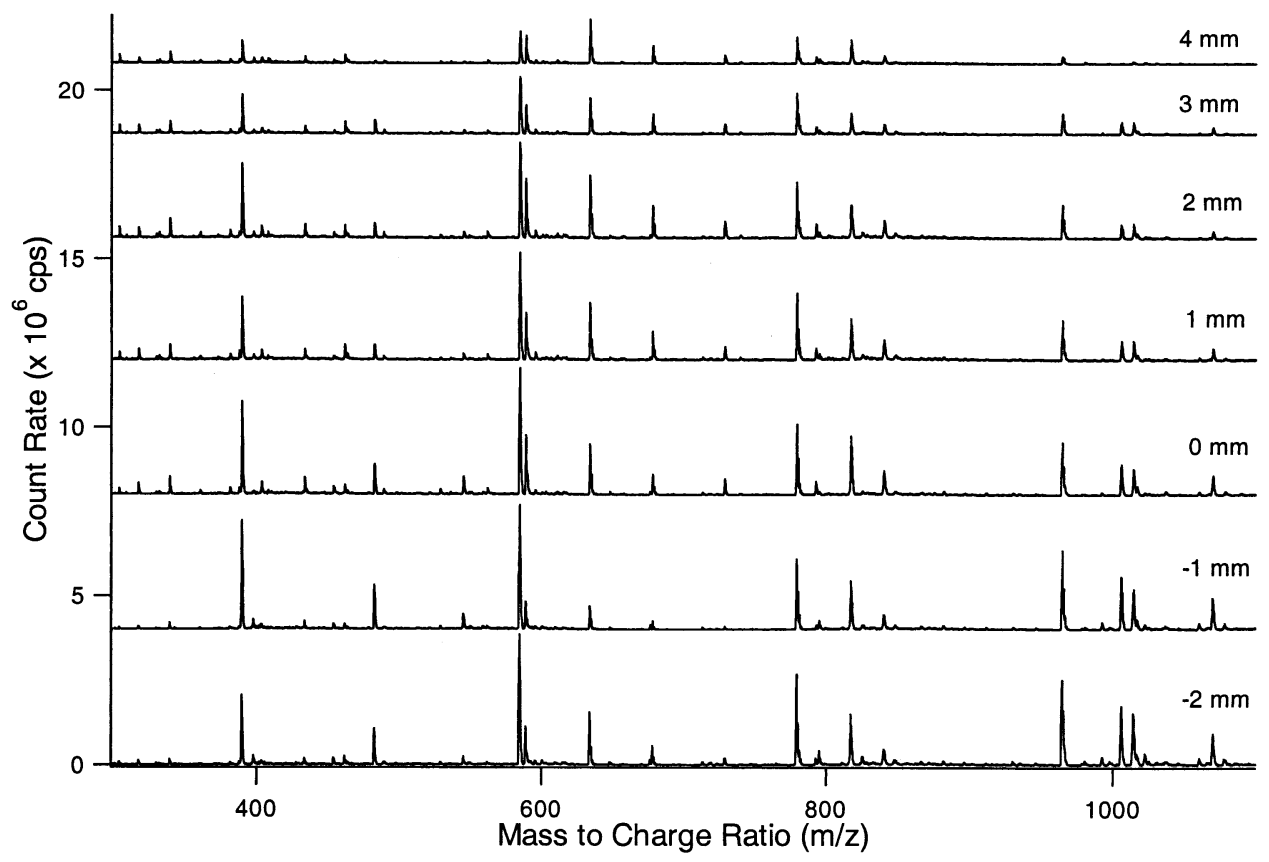

Figure 7. Comparison of nanospray mass spectra generated for a cytochrome $c$ digest with various distances between the sprayer and the curtain plate. Peaks at $\mathrm{m} / \mathrm{z} 1168,964,779,678$, and 634 correspond to singly charged peptides. Peaks at $\mathrm{m} / \mathrm{z} 817,482,390,339,317$, and 545 correspond to multiply charged peptides.

lets. Figure 7 shows data collected for a cytochrome $c$ digest (diluted in 50/50 water/methanol with $0.1 \%$ formic acid) with various spacings between a nanospray tip and the curtain plate ( -2 to $4 \mathrm{~mm}$ ). Since the curtain plate-heated chamber spacing was $3 \mathrm{~mm}$, the sprayer to chamber distance can be interpolated by addition of $3 \mathrm{~mm}$ to the values in the legend. The potentials were 500,500 , and $80 \mathrm{~V}$ applied to the curtain plate, chamber, and orifice, respectively. The curtain gas flow rate was $0.2 \mathrm{~L} / \mathrm{min}$ and no sheath gas was provided. Sample was provided to the nanospray tip with a flow rate of $50 \mathrm{~nL} / \mathrm{min}$. The electrospray potential was adjusted from $2500 \mathrm{~V}$ at the position defined as $-2 \mathrm{~mm}$ to $3300 \mathrm{~V}$ at the position defined as $4 \mathrm{~mm}$. The spectra contained peaks corresponding to a multitude of singly charged peptides including $\mathrm{m} / \mathrm{z} 1168,964,779$, 678 , and 634 , as well as multiply charged peptides corresponding to $\mathrm{m} / \mathrm{z} 817,482,390,339,317$, and 545 . The count rate for the peptides was relatively constant with the sprayer flush with or inserted into the curtain plate $(-2 \mathrm{~mm}-0 \mathrm{~mm}$ in the figure). Increasing the separation between the nanospray tip and the curtain plate over the range of $1-4 \mathrm{~mm}$ decreased the count rate for peptides from the digest. When running samples at very low flow rates with this interface, it is advantageous to insert the nanospray tip through the aperture in the curtain plate to improve the stability due to a lensing effect [3]. For this sample, there was no need to heat the chamber. However, for samples prepared with a higher aqueous content in the solvent, it was beneficial to heat the chamber to approximately $74^{\circ} \mathrm{C}$. The addition of heat provided only marginal improvement in ion count rates, but it did help to reduce the low mass chemical noise in an analogous fashion to the data shown in Figure 6.

An experiment was conducted to determine the long-term stability of the nanospray signal with this interface. A cytochrome $c$ digest was sprayed continuously for approximately $19 \mathrm{~h}$ at a flow rate of 50 $\mathrm{nL} / \mathrm{min}$. The electrospray potential was $2300 \mathrm{~V}$, but increased to $2600 \mathrm{~V}$ over the course of $19 \mathrm{~h}$ because of deterioration and salting at the tip of the sprayer. The nanospray tip was inserted up to $2 \mathrm{~mm}$ through the aperture in the curtain plate, and the position was not changed over the course of the experiment. Spectra were collected at time points 0 and $19 \mathrm{~h}$, as shown in Figure 8 . The count rate for peptides from the digest was very stable over the course of $19 \mathrm{~h}$. There was a marginal decrease in count rate for the peptides, however this was most likely a result of tip deterioration. These data demonstrate that the particle discriminator provides a robust interface for electrospray ionization at very low solution flow rates.

Current efforts are focused on using this interface to improve the sensitivity and reliability for nanoflow LC-MS by reducing the effects of solvent composition on the signal for various analytes. In addition, computational fluid dynamics (CFD) is being used to monitor the behavior of neutrals, ions, and charged particles within this interface. This should lead to an improved understanding of the mechanisms leading to charged particle discrimination. 


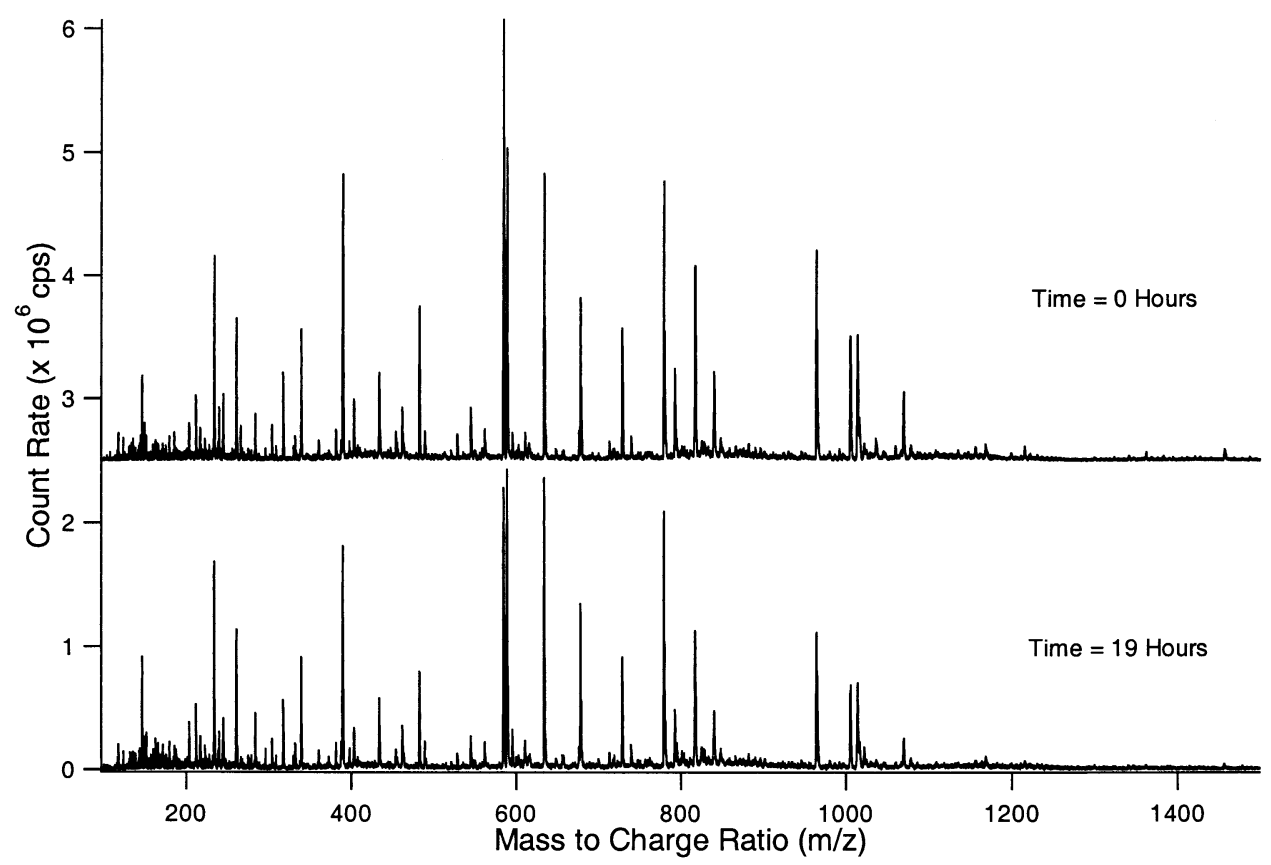

Figure 8. Comparison of nanospray spectra for a cytochrome $c$ digest initially and after $19 \mathrm{~h}$ of operation. Peaks at $\mathrm{m} / \mathrm{z} 1168,964,779,678,634,561$, and 283 correspond to singly charged peptides. Peaks at $\mathrm{m} / \mathrm{z} 817,545,482,454,433,403,390,339,317$, and 303 correspond to multiply charged peptides.

\section{Conclusions}

The data presented in this paper demonstrate that the combination of a heated laminar flow chamber, particle discriminator space, and a counter-current gas flow provide an interface that makes it possible to conduct stable electrospray ionization with solution flow rates that vary from the low $\mathrm{nL} / \mathrm{min}$ regime up to greater than $1000 \mathrm{~nL} / \mathrm{min}$. In addition, the combination of two separate chambers for desolvation (counter-current gas and heat) within the atmospheric pressure region prior to the orifice makes it possible to generate stable electrosprays with samples prepared in solvents ranging from $100 \%$ aqueous to $100 \%$ organic. The incorporation of two distinct regions for particle discrimination substantially improves the long-term stability and robustness of this interface, particularly for samples prepared in solvents containing predominantly water. In addition, the improved desolvation provided by the combination of counter-current gas flow and heat improves the count rate by approximately a factor of two for samples prepared in aqueous solutions. This interface effectively separates the processes of desolvation and gas conductance limitation to eliminate some of the sensitivity losses that typically occur in ESI-MS when analytes are prepared in aqueous solvent. The incorporation of two separate atmospheric pressure regions for desolvation improves the efficiency, and eliminates the problem of boiling solution in nanospray tips.

\section{Acknowledgments}

The authors thank Deolinda Fernandez for preparing all of the samples used in these studies. This work was supported by the Natural Sciences and Engineering Research Council of Canada (NSERC) and Applied Biosystems/MDS SCIEX. BBS acknowledges an Industrial Research Fellowship from NSERC.

\section{References}

1. Yamashita, M.; Fenn, J. B. Electrospray Ion Source. Another Variation on the Free-Jet Theme. J. Phys. Chem. 1984, 88, 4451-4459.

2. Bruins, A. P.; Covey, T. C.; Henion, J. D. Ion Spray Interface for Combined Liquid Chromatography/Atmospheric Pressure Ionization Mass Spectrometry. Anal. Chem. 1987, 59, 26422646.

3. Schneider, B. B.; Douglas, D. J.; Chen, D. D. Y. An Atmospheric Pressure Ion Lens to Improve Electrospray Ionization at Low Solution Flow-Rates. Rapid Commun. Mass Spectrom. 2001, 15, 2168-2175.

4. Janiszewski, J. S.; Rogers, K. J.; Whalen, K. M.; Cole, M. J.; Liston, T. E.; Duchoslav, E.; Fouda, H. G. A High-Capacity LC/MS System for the Bioanalysis of Samples Generated from Plate-Based Metabolic Screening. Anal. Chem. 2001, 73, 14951501.

5. Whitehouse, C. M.; Dreyer, R. N.; Yamashita, M.; Fenn, J. B. Electrospray Interface for Liquid Chromatographs and Mass Spectrometers. Anal. Chem. 1985, 57, 675-679.

6. Bateman, K. P.; White, R. L.; Thibault, P. Disposable Emitters for On-Line Capillary Zone Electrophoresis/Nanoelectrospray Mass Spectrometry. Rapid Commun. Mass Spectrom. 1997, 11, 307-315.

7. Wilm, M.; Mann, M. Analytical Properties of the Nanoelectrospray Ion Source. Anal. Chem. 1996, 68, 1-8. 
8. Crescenzi, C.; Corcia, A. D.; Marchese, S.; Sampari, R. Determination of Acidic Pesticides in Water by a Benchtop Electrospray Liquid Chromatography Mass Spectrometer. Anal. Chem. 1995, 67, 1968-1975.

9. Volmer, D. A.; Brombacher, S.; Whitehead, B. Studies on Azaspiracid Biotoxins. I. Ultrafast High-Resolution Liquid Chromatography/Mass Spectrometry Separations using Monolithic Columns. Rapid Commun. Mass Spectrom. 2002, 16, 2298-2305.

10. Chowdhury, S. K., Katta, V., Chait, B. T. United States Patent \#4977320. Electrospray Ionization Mass Spectrometer with New Features; Jan. 22, 1990.

11. Kirby, D. P.; Thorne, J. M.; Gotzinger, W. K.; Karger, B. L. CE/ESI-MS Interface for Stable, Low-Flow Operation. Anal. Chem. 1996, 68, 4451-4457.

12. Tomany, M. J., Jarrell, J. A. United States Patent \#5304798.
Housing for Converting an Electrospray to an Ion Stream; Apr. 19 1994.

13. Olivares, J. A.; Nguyen, N. T.; Yonker, C. R.; Smith, R. D. On-Line Mass Spectrometric Detection for Capillary Zone Electrophoresis. Anal. Chem. 1987, 59, 1230-1232.

14. Tanner, S. D.; Douglas, D. J.; French, J. B. Gas and Ion Dynamics of a Three-Aperture Vacuum Interface for Inductively Coupled Plasma-Mass Spectrometry. App. Spectrosc. 1994, 48, 1373-1378.

15. Lin, B.; Sunner, J. Ion Transport by Viscous Gas Flow Through Capillaries. J. Am. Soc. Mass Spectrom. 1994, 5, 873-885.

16. Konenkov, N. V.; Korolkov, A. N.; Stepanov, V. A. J. Tech. Phys. 1996, 66, 176-180 (Russ.).

17. Revercomb, H. E.; Mason, E. A. Theory of Plasma Chromatography/Gaseous Electrophoresis-A Review. Anal. Chem. 1975, 47, 970-983. 